\title{
Cardiac Sarcoidosis: F18FDG-PET/CT as the Most Helpful Tool in the Decision Making of Medical Management, and Assessment of Response to Treatment
}

\author{
Sana S Khan ${ }^{1 *}$, Michael Lafemina ${ }^{2}$, Cameron Kroll ${ }^{1}$, Manreet Singh ${ }^{1}$, Tianye Liu ${ }^{1}$ Sukhkarn N Bains ${ }^{1}$, and Carina Mari Aparici ${ }^{1,3}$ \\ ${ }^{1}$ Department of Radiology and Center for Molecular and Functional Imaging (CMFI) at China Basin, University of California, San Francisco, USA \\ ${ }^{2}$ San Francisco Veterans Affairs, Medical Service, Pulmonary, Critical Care, and Sleep Section, USA \\ ${ }^{3}$ Department of Radiology, San Francisco Veterans Affairs, Nuclear Medicine Division, USA
}

\begin{abstract}
We describe the case of a 32-year-old healthy man who presented to the Emergency Department (ED) with vomiting, dizziness, and diaphoresis in addition to transient episodes of loss of consciousness on the day of admission. The patient's past medical history was unremarkable. Once in the ED triage vitals showed a heart rate of 254 beats per minute and an initial Electrocardiogram (EKG) showing stable monomorphic ventricular tachycardia. After conversion to sinus rhythm the patient was admitted where and after a long array of tests, the patient was diagnosed with cardiac sarcoidosis. Implantable Cardioverter-Defibrillators (ICD) was implanted and he was started on corticosteroids. The medical management of the patient's treatment with corticosteroids was very challenging. Fludeoxyglucose positron emission tomography/ computed tomography (FDG-PET/CT) was the most helpful tool and the test of choice for the follow up in his medical management.
\end{abstract}

\section{Keywords: FDG; PET/CT; Cardiac sarcoidosis management}

\section{Introduction}

Sarcoidosis is a chronic inflammatory disease of unknown cause that affects multiple organs and is characterized histologically by noncaseating granulomas in affected tissues. The occurrence of sarcoidosis varies greatly by ethnicity; between 10 to 20 out of 100,000 Caucasians are affected annually compared to 35 out of 100,000 African Americans per year [1]. Sarcoidosis generally has good prognosis, high remission rate, and low mortality [2]. However, cardiac involvement significantly affects diagnosis, management, and survival. Involvement of the myocardium is present in at least $25 \%$ of patients with sarcoidosis, which accounts for as many as $13-25 \%$ of deaths from the disease [3]. The current clinical diagnosis for cardiac sarcoidosis is established by the Japanese Ministry of Health and Welfare Diagnostic Guidelines [4]. These guidelines require either direct biopsy-proven confirmation of cardiac sarcoidosis or direct histologically proven extra cardiac sarcoidosis combined with indirect evidence of an inflammatory myocardial lesion. Confirmation of cardiac sarcoidosis by endomyocardial biopsy is highly specific. However, biopsy is hindered by reduced sensitivity in the range of $20 \%$ due to the patchy nature of myocardial involvement and the selective nature of sampling which often targets the apical septum. Indirect evidence of cardiac sarcoidosis includes criteria such as uptake of the tracer Gallium-67, myocardial scarring resulting in a perfusion defect, and functional impairment of myocardial wall motion or conduction abnormalities [5]. Clinical manifestations of cardiac sarcoidosis range from asymptomatic disease to life threatening arrhythmias, conduction abnormalities, and congestive heart failure. Due to the unpredictable nature that comes with arrhythmias, many patients often have the insertion of permanent pacemakers or implanted cardioverter-defibrillators (ICD).

Corticosteroid therapy (CS) is the primary therapy of choice for cardiac sarcoidosis. Early initiation of CS therapy has been associated with improvement, if not complete response. Standard treatment for initial CS therapy is high doses of prednisone in the range of 60-80 $\mathrm{mg} /$ day [6]. Due to the possible fatal outcome of cardiac sarcoidosis, long-term maintenance therapy is usually required. Adverse effects of CS include hypertension, headaches, osteoporisis and the development of ventricular aneurysms. Methotrexate, azathioprine, hydroxychloroquine, and cyclyphophamide used concurrently with prednisone have had varying efficacy $[7,8]$. The degree of inflammatory activity and systemic distribution of the disease helps in determining appropriate treatment. The TNF- $\alpha$ inhibitor, infliximab, has also been reported in the literature as a successful treatment for cardiac sarcoidosis [9]. The duration of CS therapy is poorly defined in the literature, and recurrence is usually observed with tapering or cessation of medication [10]. Furthermore, there is no established reliable technique to help in the decision making of medical management, and accurate assessment of response to treatment versus recurrence.

FDG-PET/CT is widely used in the characterization, staging, restaging and surveillance of multiple malignancies. It can also play a major role in the diagnosis of patients with suspected infection and inflammatory changes [11]. Activated inflammatory cells have a higher glycolitic rate, and therefore increase their expression of glucose transporters and the uptake of FDG. In sarcoidosis, macrophages are activated while involved in the formation of granulomas, which explain why the granulomatous lesions of sarcoidosis show strong FDG activity. In sarcoidosis, the degree of FDG uptake has been related both to activity of disease [11] and to treatment responsiveness [12].

We present the case of a young healthy man diagnosed with cardiac sarcoidosis. He was placed on CS and had a complicated clinical management with multiple flairs and recurrences. FDG-PET/CT was the most helpful technique for the assessment of treatment response/ recurrence in the heart as well as in the rest of the body. Although

${ }^{*}$ Corresponding author: Sana Khan, Department of Radiology and Center for Molecular and Functional Imaging (CMFI) at China Basin, University of California, San Francisco, USA, Tel: 408-799-3353; Fax: 415-353-8571 E-mail: sana.khan@ucsf.edu

Received September 05, 2013; Accepted October 25, 2013; Published October 28, 2013

Citation: Khan SS, Lafemina M, Kroll C, Singh M, Liu T, et al. (2013) Cardiac Sarcoidosis: F18FDG-PET/CT as the Most Helpful Tool in the Decision Making of Medical Management, and Assessment of Response to Treatment. J Pulm Respir Med 3: 160. doi:10.4172/2161-105X. 1000160

Copyright: ( 2013 Khan SS, et al. This is an open-access article distributed under the terms of the Creative Commons Attribution License, which permits unrestricted use, distribution, and reproduction in any medium, provided the original author and source are credited. 
other papers have described the use of FDG-PET in the diagnoses of sarcoidosis in different organs, including the myocardium, we believe we present for the first time the use of FDG-PET/CT as the technique of choice to guide the complicated medical management of cardiac sarcoidosis.

\section{Diagnosis}

We present the case of a 32-year old male admitted to the ED with vomiting, dizziness, chest discomfort, shortness of breath, and mild tingling in both hands; claiming to have become diaphoretic with syncopal episodes two and a half hours prior to admittance. The patient had unremarkable prior medical history but family history of multiple myocardial infarctions. The patient denied a history of heavy alcohol usage or illicit drugs. After being admitted to the ED he developed dizziness. An EKG showed wide-complex tachycardia with a rate of 255. Labs showed troponin levels at 0.85 , which is classified as high. His systolic blood pressure was in the $80 \mathrm{~s}$, all these factors pointed to the need for an immediate external cardioversion with a single shock of 200J, which resulted in the successful restoration of his sinus rhythm.

The patient was then admitted to the Critical Care Unit (CCU) ward for further evaluation and management. Echocardiography revealed a decreased ejection fraction of 40-45 percent as well as mitral and tricuspid regurgitation. Coronary angiogram was unremarkable; doctors felt follow for congestive heart failure was not necessary unless the patient developed signs or symptoms of heart failure. Myocardial perfusion demonstrated abnormal perfusion in a patchy pattern that did not follow the coronary distribution. A chest CT showed enlarged mediastinal lymph nodes and ill defined lingular opacities that were nonspecific. A cardiac MRI with gadolinium revealed a patchy pattern or gadolinium enhancement involving the RV, septum and inferoseptum consistent with an inflammatory process such as myocarditis, sarcoidosis or amyloidosis. Ocular examination did not show evidence of uveitis.

Initial PFT values were as follows: FEV1:3.98; FVC: 4.74; TLC: 6.24. Subsequent PFTs demonstrated significant deterioration of these values ie: FEV1: 3.62; FVC: 4.35; TLC: 5.19.

While in the CCU the patient was received $400 \mathrm{mg}$ amiodarone and $25 \mathrm{mg}$ of metoprolol. However, due to the intermittent nature of his ventricular arrhythmias, an ICD was placed. Patient underwent bronchoscopy with transbronchial biopsy, which was non-diagnostic. Soon after the patient then underwent a mediastinoscopy with lymph node biopsy that showed non-caseasting granulomas and he was diagnosed with sarcoidosis with cardiac involvement.

\section{Clinical Management}

The patient was started on $60 \mathrm{mg}$ of oral prednisone daily. The dosage was decreased over four months until $40 \mathrm{mg} /$ day. At this point, an assessment of treatment response was recommended for monitoring disease activity in accordance to dose adjustment. After the patient had been on steroid therapy for four months the first FDG-PET/CT was requested this time instead of an MRI for evaluation of response to treatment by patient's cardiac sarcoidosis. Dedicated cardiac and whole body FDG-PET/CT images showed abnormal mildly increased FDG activity in the septum and inferior left ventricular wall in a geographic/ patchy patter. These findings were concordant with prior findings from the cardiac MRI, indicating persistent active myocardial sarcoidosis. Additionally, focal FDG activity was observed at the right acetabulum and right scapula, consistent with bony involvement of sarcoid. [DEXA scan showed the patient was defined as osteopenic based on the ISCD criteria with a PA lumbar spine total value of -1.5 . A chest $\mathrm{x}$-ray revealed clear lungs.] Based on the FDG-PET/CT findings, patient's predisone dosage was kept at 40mg daily for 3 more months until next FDG-PET/ CT was scheduled.

A second FDG-PET/CT scan was performed 3 months later to assess patient's response to treatment and depicted a similar unchanged distribution and level of FDG activity. [Based on these findings, the patient's prednisone was then tapered down to $20 \mathrm{mg}$ with continued close clinical monitoring. Unfortunately, a subsequent PET/CT demonstrated significant interval worsening of disease activity with increased involvement of the myocardium, bones, and lymph nodes. Based on radiographic worsening, prednisone dose was increased and methotrexate was initiated. Despite these changes in medical therapy, a subsequent $\mathrm{PET} / \mathrm{CT}$ three months later again demonstrated interval worsening of the disease with a marked interval increase in metabolic activity of known sites and new/progressive involvement of the myocardium, supraclavicular, mediastinal, hilar, retroperitoneal, and mesenteric lymph nodes. Ultimately the decision was made to initiate $400 \mathrm{mg}$ of infliximab given disease significant radiographic progression despite corticosteroid therapy and methotrexate. Follow up PET/CT imaging 3 months after initiation of infliximab demonstrated interval improvement in cardiac, mediastinal, peripancreatic, mesenteric, and bony sarcoid lesions consistent with treatment response. Follow up PET/ CT scan obtained four months later finally showed interval response to treatment with improvement in the myocardium, lymphadeopathy , and bony lesions. Subsequently, the patient has been weaned off of prednisone and remains on methotrexate and infliximab. His most recent PET/CT scan is without any evidence of active sarcoid.

\section{FDG-PET/CT Imaging Methods}

The FDG-PET/CT studies were obtained using a Discovery 64 slice PET/CT scanner GE 690 with LYSO crystals and time of flight.

A strict low carbohydrate diet was followed for 48 hours before the study to minimize nonspecific cardiac activity. The patient was NPO for 6 hours prior to the test. After assessment of glucose levels, the patient was intravenously administered with $8 \mathrm{mCi}(370 \mathrm{MBq})$ of F18-FDG in the antecubital region using a MEDRAD INTEGO robot. Sixty minutes later, he was taken to the scanner for imaging. A transmission scan (5 mm contiguous axial cuts) was obtained using an integrated multi-slice helical non-enhanced CT from vertex to toes for attenuation correction and anatomic localization. The acquisition was obtained with a oneslice overlap at the borders of the field of view to avoid artifacts, using $120 \mathrm{kV}, 75 \mathrm{mAs}$, and a $512 \times 512$ matrix size. Immediately after, and without moving the patient, and emission scan was obtained in $3 \mathrm{D}$ mode with time of flight in 11 beds at 3 minutes per bed over the same anatomical regions. The PET emission scan was corrected using segmented attenuation data of the conventional transmission scan. A Gaussian filtering $(6.4 \mathrm{~mm})$ was performed for smoothing of images. The PET images were reconstructed with a standard iterative algorithm (OSEM, two iterative steps, 24 subsets) using GE software release 5.0 VUE Point FX intelligent reconstructions (Figure 1).

\section{Discussion and Review of Literature}

Presentation of cardiac involvement is unpredictable, ranging from silent disease to sudden cardiac death from ventricular arrthymia. In addition to classic symptoms of systematic sarcoidosis such as fever, weight loss and malaise, common cardiac symptoms include progressive dysnea, fatigue, palpitations and syncope [13]. Complete heart block is the most common finding in patients with evident cardiac sarcoidosis and is reportly in $23-30 \%$ of patients [14]. There 

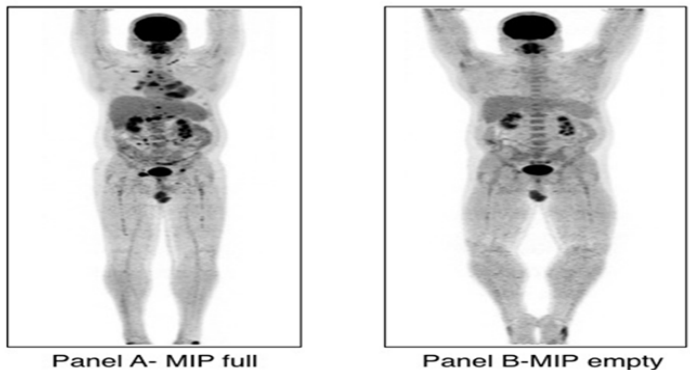

FDG-PET/CT MIP (Maximum Intensity Projection) images of the same patien during initial treatment (panel A) and after changing treatment (panel B). Pane A shows intensely hypermetabolic sarcoid disease in the heart, bones and lymph nodes while the patient was on treatment with steroids. Panel B shows complete response to new treatment

Figure 1: FDG-PET/CT Imaging.

have also been reported symptoms that include anginal chest pain mimicking acute coronary syndromes and pleuritic chest pains from pericardial involvement [15]. Acute pericarditis, pericardial effusions, and rarely tamponade have been reported manifestations of cardiac sarcodosis [16,17]. It has been documented that cardiac sarcoidosis frequently presents as Dilated Cardiomyopathy (DCM). Patients initially diagnosed with end-stage DCM were rediagnosed with cardiac sarcoidosis upon histological examination of the re sected myocardium $[18,19]$. What made the diagnosis so difficult were the patient's age, history and initial symptoms. When the patient first presented to the emergency department his initial vitals, VT, right bundle-type pattern and EKG were typically seen in someone with arrhytmogenic ventricular dyslasia/cardiomyoparthy (ARVD) or Brugada's syndrome, which causes ventricular arrest without preceding symptoms, in men of his age. After a CT was taken it still was not clear what he exactly had because he presented with finding one would see in diseases such as myocarditis and amyloidosis

Enhanced Cardiac Magnetic Resonance imaging (CMR) with gadolinium-diethylenetricamine pentaacetic acid enhancement has shown to be helpful in the detection of myocardial sarcoidosis [20]. It seems to be possible to track disease activity and response treatment of cardiac sarcoidosis with CMR [21]. Contrast- enhanced Cardiac Magnetic Resonance imaging (CMR) with gadoliniumdiethylenetriamine penta-acetic acid accu- rately identifies myocardial scarring in ischaemic heart disease with a high degree of spatial accuracy. Cardiac MRI also enables high-resolution evaluation of cardiac function and struc- ture at the time of assessment. The early involvement of myocardium not apparent on ECG or clinical findings can therefore be detected on CMR [22,23]. However, CMR is limited in its use in patients with pacemakers, defibrillators, and mechanical valves [24]. This was the case in our patient; he was able to have an MRI in the diagnostic stage of his disease, but due to the consistent nature of his ventricular tachycardia an ICD was needed. Soon after his ICD placement, the diagnosis of cardiac sarcoidosis was confirmed from his biopsy resulting in a need for a different imaging modality, other than CMR, to monitor his CS therapy response. Patients with ICD placement create a challenge to image cardiac sarcoidosis with MRI however, there is an alternative imagining modality that acts as an excellent tool in the follow up treatment of the disease and also provides assessment of the whole body.

PET scanning using 18F-fluorodeoxyglucose (FDG) has been used to identify areas of active inflammation. The basis of 18-FDG
PET imaging is that inflammatory cells have increased uptake or accumulation of FDG. Uptake of radiotracers show a relationship with disease activity and correspondingly, a decrease in uptake of FDG may be seen with corticosteroid therapy (CS) [25]. Yamagishi and colleagues showed that $18 \mathrm{~F}-\mathrm{FDG}$ PET accumulates in the myocardium of patients with cardiac sarcoidosis and that 18F-FDG PET shows higher sensitivity and for the detection of myocardial involvement compared to thallium-201 SPECT and gallium-67 scintigraphy [26]. According to the Stanford Dosimetry RADAR radiation calculator, the amount of radiation the patient received per scan was about $7.03 \mathrm{mSv}$ or 703 mrem for the whole body, which is approximately equivalent to a whole body exposure of 855 days (2.343 years) of exposure to natural background radiation. According to the National Institute of Health (NIH) three-tier system the effective doses between $3 \mathrm{mSv}$ (300 mrem) and $50 \mathrm{mSv}$ (5000 mrem, or 5 rem), the risk is considered "minimal was necessary [27] for this patient's medical management. We were able to use 18F-FDG PET in the patient's treatment plan through monitoring his response to CS therapy. The patient was started off on high levels of CS and gradually tapered down in concordance to his flares, which were observed quarterly through the use of 18F-FDG PET. By monitoring disease activity through FDG/PET in this patient, corticosteroid therapy has been weaned off and an effective regimen of methotrexate and remicaid has been identified. On this therapy, there is no evidence of disease activity and side effects of chronic corticosteroid use can be avoided.

\section{References}

1. Rybicki BA, Major M, Popovich J Jr, Maliarik MJ, lannuzzi MC (1997) Racia differences in sarcoidosis incidence: a 5-year study in a health maintenance organization. Am J Epidemiol 145: 234-241.

2. Ayyala US, Nair AP, Padilla ML (2008) Cardiac sarcoidosis. Clin Chest Med 29: 493-508, ix.

3. Silverman KJ, Hutchins GM, Bulkley BH (1978) Cardiac sarcoid: clinicopathologic study of 84 unselected patients with systemic sarcoidosis. Circulation 58: 1204-1211.

4. Mc Ardle BA, Leung E, Ohira $H$, Cocker MS, deKemp RA, et al. (2013) The role of $F(18)$-fluorodeoxyglucose positron emission tomography in guiding diagnosis and management in patients with known or suspected cardiac sarcoidosis. J Nucl Cardiol 20: 297-306.

5. Youssef G, Beanlands RS, Birnie DH, Nery PB (2011) Cardiac sarcoidosis: applications of imaging in diagnosis and directing treatment. Heart 97: 20782087.

6. Shabetai R (2000) Sarcoidosis and the Heart. Curr Treat Options Cardiovasc Med 2: 385-398.

7. Chapelon-Abric C, de Zuttere D, Duhaut P, Veyssier P, Wechsler B, et al. (2004) Cardiac sarcoidosis: a retrospective study of 41 cases. Medicine (Baltimore) 83: 315-334

8. Demeter SL (1988) Myocardial sarcoidosis unresponsive to steroids. Treatment with cyclophosphamide. Chest 94: 202-203.

9. Uthman I, Touma Z, Khoury M (2007) Cardiac sarcoidosis responding to monotherapy with infliximab. Clin Rheumatol 26: 2001-2003.

10. Sekiguchi M, Yazaki Y, Isobe M, Hiroe M (1996) Cardiac sarcoidosis: diagnostic prognostic, and therapeutic considerations. Cardiovasc Drugs Ther 10: 495-510.

11. Zhuang H, Alavi A (2002) 18-fluorodeoxyglucose positron emission tomographic imaging in the detection and monitoring of infection and inflammation. Semin Nucl Med 32: 47-59

12. Yamada Y, Uchida Y, Tatsumi K, Yamaguchi T, Kimura H, et al. (1998) Fluorine-18-fluorodeoxyglucose and carbon-11-methionine evaluation of lymphadenopathy in sarcoidosis. J Nucl Med 39: 1160-1166.

13. Chapelon-Abric C, de Zuttere D, Duhaut P, Veyssier P, Wechsler B, et al. (2004) Cardiac sarcoidosis: a retrospective study of 41 cases. Medicine (Baltimore) 83: 315-334.

14. Roberts WC, McAllister HA Jr, Ferrans VJ (1977) Sarcoidosis of the heart. 
Citation: Khan SS, Lafemina M, Kroll C, Singh M, Liu T, et al. (2013) Cardiac Sarcoidosis: F18FDG-PET/CT as the Most Helpful Tool in the Decision Making of Medical Management, and Assessment of Response to Treatment. J Pulm Respir Med 3: 160. doi:10.4172/2161-105X. 1000160

Page 4 of 4

A clinicopathologic study of 35 necropsy patients (group 1) and review of 78 previously described necropsy patients (group 11). Am J Med 63: 86-108.

15. Wait JL, Movahed A (1989) Anginal chest pain in sarcoidosis. Thorax 44: 391-395.

16. Kinney E, Murthy R, Ascunce G, Donohoe R, Zelis R (1979) Pericardial effusions in sarcoidosis. Chest 76: 476-478.

17. Verkleeren JL, Glover MU, Bloor C, Joswig BC (1983) Cardiac tamponade secondary to sarcoidosis. Am Heart J 106: 601-603.

18. Terasaki F, Ukimura A, Tsukada B, Fujita S, Katashima T, et al. (2008) Enhanced expression of type 1 helper T-cell cytokines in the myocardium of active cardiac sarcoidosis. Circ J 72: 1303-1307.

19. Roberts WC, Vowels TJ, Ko JM, Capehart JE, Hall SA (2009) Cardiac transplantation for cardiac sarcoidosis with initial diagnosis by examination of the left ventricular apical "core" excised for insertion of a left ventricular assist device for severe chronic heart failure. Am J Cardiol 103: 110-114.

20. Futamatsu H, Suzuki J, Adachi S, Okada H, Otomo K, et al. (2006) Utility of gallium-67 scintigraphy for evaluation of cardiac sarcoidosis with ventricular tachycardia. Int J Cardiovasc Imaging 22: 443-448.

21. Schulz-Menger J, Wassmuth R, Abdel-Aty H, Siegel I, Franke A, et al. (2006)
Patterns of myocardial inflammation and scarring in sarcoidosis as assessed by cardiovascular magnetic resonance. Heart 92: 399-400.

22. Kim RJ, Fieno DS, Parrish TB, Harris K, Chen EL, et al. (1999) Relationship of MRI delayed contrast enhancement to irreversible injury, infarct age, and contractile function. Circulation 100: 1992-2002.

23. Smedema JP, Snoep G, van Kroonenburgh MP, van Geuns RJ, Dassen WR, et al. (2005) Evaluation of the accuracy of gadolinium-enhanced cardiovascular magnetic resonance in the diagnosis of cardiac sarcoidosis. J Am Coll Cardiol 45: $1683-1690$.

24. Doughan AR, Williams BR (2006) Cardiac sarcoidosis. Heart 92: 282-288.

25. Teirstein AS, Machac J, Almeida O, Lu P, Padilla ML, et al. (2007) Results of 188 whole-body fluorodeoxyglucose positron emission tomography scans in 137 patients with sarcoidosis. Chest 132: 1949-1953.

26. Yamagishi H, Shirai N, Takagi M, Yoshiyama M, Akioka K, et al. (2003) Identification of cardiac sarcoidosis with (13) N-NH(3)/(18)F-FDG PET. J Nuc Med 44: 1030-1036.

27. Stabin (1997) M RADAR medical procedure radiation dose calculator and consent language generator. Stanford dosimetry, LLC. 